TRANSACTIONS OF THE

AMERICAN MATHEMATICAL SOCIETY

Volume 355, Number 9, Pages 3463-3474

S 0002-9947(03)03288-4

Article electronically published on May 29, 2003

\title{
ANDERSON'S DOUBLE COMPLEX AND GAMMA MONOMIALS FOR RATIONAL FUNCTION FIELDS
}

\author{
SUNGHAN BAE, ERNST-ULRICH GEKELER, PYUNG-LYUN KANG, \\ AND LINSHENG YIN
}

\begin{abstract}
We investigate algebraic $\Gamma$-monomials of Thakur's positive characteristic $\Gamma$-function, by using Anderson and Das' double complex method of computing the sign cohomology of the universal ordinary distribution. We prove that the $\Gamma$-monomial associated to an element of the second sign cohomology of the universal ordinary distribution of $\mathbb{F}_{q}(T)$ generates a Kummer extension of some Carlitz cyclotomic function field, which is also a Galois extension of the base field $\mathbb{F}_{q}(T)$. These results are characteristic- $p$ analogues of those of Deligne on classical $\Gamma$-monomials, proofs of which were given by Das using the double complex method. In this paper, we also obtain some results on $e$-monomials of Carlitz's exponential function.
\end{abstract}

\section{INTRODUCTION}

In [An1] Anderson invented a remarkable method of computing in an identical way the sign cohomology of the universal ordinary distributions, both for the rational number field and a global function field. He introduced a certain double complex which is a resolution of the universal ordinary distribution. This double complex enabled him to construct canonical basis classes of the sign cohomology. Das [Da used this double complex in the rational number field case for the study of classical $\Gamma$-monomials and got a series of results, which greatly illuminated the power of Anderson's method.

In this paper, using Anderson's double complex and following Das' method, we study $\Gamma$-monomials for rational function fields. Thakur [Th] defined the $\Gamma$ function in characteristic $p$ and showed that it has many interesting properties analogous to the classical $\Gamma$-function. Especially, it satisfies a reflection formula and a multiplication formula. Sinha [Si] used Anderson's soliton theory to develop an analogue of Deligne's reciprocity for function fields. In the course of this he found that certain $\Gamma$-monomials generate Kummer extensions of cyclotomic function fields, a result which will be reproved below with the aid of the double complex. Using $\Gamma$-monomials we also find extensions of cyclotomic function fields, and these happen to be Galois even over the basic rational function field.

We would like to emphasize the following technical points: Besides the double complex, there are several main ingredients in computing the $\Gamma$-monomials in Das'

Received by the editors March 12, 2001.

2000 Mathematics Subject Classification. Primary 11R58.

The first author was supported by KOSEF cooperative Research Fund and DFG.

The fourth author was supported by Distinguished Young Grant in China and a fund from Tsinghua.

(C)2003 American Mathematical Society 
paper, and these are used frequently. In the case of a rational function field there are more roots of unity, which causes the definitions of the vertical shift operator and "canonical lifting operator" to be more complicated. In addition, the reflection formula and the multiplication formula of the $\Gamma$-function play important roles in our study. These formulae in the function field case have some extra factors, and thus one has to be more careful in applying them.

\section{The Double COMPlex for $\mathbb{F}_{q}(T)$}

Let $K=\mathbb{F}_{q}(T)$ and $A=\mathbb{F}_{q}[T]$, the rational function field and polynomial ring, respectively, over the finite field $\mathbb{F}_{q}$. We fix a generator $\gamma$ of $J=\mathbb{F}_{q}^{*}$. Let $\mathcal{A}$ be the free abelian group generated by symbols $[a]$ with $a \in K / A$. Let $\mathbb{U}$ be the quotient of $\mathcal{A}$ by the subgroup generated by all elements $[a]-\sum_{\mathfrak{n} b=a}[b]$, where $\mathfrak{n}$ is a monic polynomial in $A$, and $\mathbb{U}^{-}$(resp. $\mathbb{U}^{+}$) the quotient of $\mathcal{A}$ by the subgroup generated by all elements $[a]-\sum_{\mathfrak{n} b=a}[b]$, along with all the $\sum_{\theta \in J}[\theta a]$ (resp. $[a]-[\gamma a]$ ). We call the group $\mathbb{U}$ the universal ordinary distribution on $K / A$. Further, $J$ acts on $\mathbb{U}$ in the natural way. Let $H^{*}(J, \mathbb{U})$ denote the sign cohomology group for $\mathbb{U}$. It is known that $\operatorname{tor}\left(\mathbb{U}^{+}\right) \simeq H^{1}(J, \mathbb{U})$ and $\operatorname{tor}\left(\mathbb{U}^{-}\right) \simeq H^{2}(J, \mathbb{U})([\mathrm{BGY}$, Proposition 2.4). If $\mathbf{a}=\sum m_{i}\left[a_{i}\right] \in \mathcal{A}$ represents an element in $H^{*}(J, \mathbb{U})$, we often write $\mathbf{a} \in H^{*}(J, \mathbb{U})$. It is clear from the context whether elements of $\mathcal{A}, \mathbb{U}, H^{1}(J, \mathbb{U})$, or $H^{2}(J, \mathbb{U})$ are intended. We use gothic letters to denote elements of $A$. Define

$$
\left\langle\frac{\mathfrak{a}}{\mathfrak{f}}\right\rangle= \begin{cases}1, & \text { if } \mathfrak{a} \text { is monic } \\ 0, & \text { otherwise }\end{cases}
$$

assuming that $\operatorname{deg} \mathfrak{a}<\operatorname{deg} \mathfrak{f}$ and that $\mathfrak{f}$ is monic. For $\mathbf{a}=\sum m_{i}\left[a_{i}\right] \in \mathcal{A}$ we define the total sum $T S(\mathbf{a})$ and internal sum $I S(\mathbf{a})$ of a by $\sum m_{i}$ and by $I S(\mathbf{a})=\sum m_{i}\left\langle a_{i}\right\rangle$, respectively. Let $\mathfrak{f}$ be the least common multiple of the denominators of the $a_{i}$ and let $\mathfrak{t} \in(A / \mathfrak{f})^{*}$. We define $\mathbf{a}^{\mathfrak{t}}$ by

$$
\mathbf{a}^{\mathfrak{t}}=\sum m_{i}\left[\mathfrak{t} a_{i}\right]
$$

Let $\mathcal{P}$ be the set of all monic irreducible polynomials in $A$. We fix a linear order "<" on $\mathcal{P}$. Let

$$
\mathcal{S}=\{[a, \mathfrak{g}, n]: a \in K / A, \mathfrak{g} \text { a squarefree monic polynomial, } n \text { an integer }\} .
$$

We denote by $|\mathfrak{g}|$ the number of monic irreducible polynomials dividing $\mathfrak{g}$. We define a double complex $\mathbb{S K}$ as follows: $\mathbb{S K}_{m, n}=$ the free abelian group generated by the symbols $[a, \mathfrak{g}, n] \in \mathcal{S}$ with $m=|\mathfrak{g}|$. The chain maps $\partial$ and $\delta$ of bidegree $(-1,0)$ and $(0,-1)$, respectively, are defined by

$$
\partial[a, \mathfrak{g}, n]=\sum_{i=1}^{|\mathfrak{g}|}(-1)^{i-1}\left(\left[a, \mathfrak{g} / \mathfrak{p}_{i}, n\right]-\sum_{\mathfrak{p}_{i} b=a}\left[b, \mathfrak{g} / \mathfrak{p}_{i}, n\right]\right),
$$

where $\mathfrak{g}=\mathfrak{p}_{1} \cdots \mathfrak{p}_{m}$ with $\mathfrak{p}_{i}<\mathfrak{p}_{j}$ for $i<j$, and

$$
\delta[a, \mathfrak{g}, n]= \begin{cases}(-1)^{m} \sum_{i=0}^{q-2}\left[\gamma^{i} a, \mathfrak{g}, n-1\right], & \text { for } n \text { odd } \\ (-1)^{m}([a, \mathfrak{g}, n-1]-[\gamma a, \mathfrak{g}, n-1]), & \text { for } n \text { even }\end{cases}
$$

Then it is easy to see that

$$
\partial^{2}=0, \quad \delta^{2}=0 \quad \text { and } \quad \delta \partial+\partial \delta=0 .
$$


Let $(T(\mathbb{S K}), \partial+\delta)$ be the total complex of $\mathbb{S K}$. We use the same notation $\mathbb{S K}$ for the total complex when the meaning is evident.

Let $\mathbb{S K}^{\prime}$ be the subcomplex of $\mathbb{S K}$ generated by the elements $\beta(a, n)[a, \mathfrak{g}, n]$, where

$$
\beta(a, n)= \begin{cases}q-1, & \text { if } a=0 \text { and } n \text { is even, } \\ 1, & \text { otherwise. }\end{cases}
$$

Then following the method employed by Ouyang in $\mathrm{Ou}$, we have:

Proposition 1. Let $\mathbb{U}$ be the universal ordinary distribution on $K / A$. There exist canonical isomorphisms

$$
H^{2}(J, \mathbb{U})=H_{0}\left(H_{0}(\mathbb{S K}, \partial), \delta\right)=H_{0}(\mathbb{S K}, \partial+\delta)=H_{0}\left(\mathbb{S K} / \mathbb{S K}^{\prime}, \partial+\delta\right)
$$

and

$$
H^{1}(J, \mathbb{U})=H_{-1}\left(H_{0}(\mathbb{S K}, \partial), \delta\right)=H_{-1}(\mathbb{S K}, \partial+\delta)=H_{-1}\left(\mathbb{S K} / \mathbb{S K K}^{\prime}, \partial+\delta\right) .
$$

Since

$$
\delta([0, \mathfrak{g}, n])= \begin{cases}(-1)^{n}(q-1)[0, \mathfrak{g}, n-1], & \text { if } n \text { is odd, } \\ 0, & \text { if } n \text { is even, }\end{cases}
$$

and $\partial([0, \mathfrak{g}, n])$ lies in $\mathbb{S K}^{\prime}$, we have:

Proposition 2. Given a square-free monic polynomial $\mathfrak{g}$ with $|\mathfrak{g}|=i$, we define

$$
k_{\mathfrak{g}}= \begin{cases}{[0, \mathfrak{g},-i] \in \mathbb{S K}_{i,-i} / \mathbb{S K}_{i,-i}^{\prime},} & \text { for } i \text { even }, \\ {[0, \mathfrak{g},-i-1] \in \mathbb{S K}_{i,-i-1} / \mathbb{S K}_{i,-i-1}^{\prime},} & \text { for } i \text { odd. }\end{cases}
$$

Then the collection $\left\{k_{\mathfrak{g}} ;|\mathfrak{g}|\right.$ even $\}$ (resp. $\left\{k_{\mathfrak{g}} ;|\mathfrak{g}|\right.$ odd $\}$ ) forms a $\mathbb{Z} /(q-1)$-basis for $H_{0}\left(\mathbb{S K} / \mathbb{S K K}^{\prime}, \partial+\delta\right)\left(\right.$ resp. $\left.H_{-1}\left(\mathbb{S K K} / \mathbb{S K K}^{\prime}, \partial+\delta\right)\right)$. The $k_{\mathfrak{g}}$ are referred to as canonical basis classes.

Define the vertical shift operator $S: \mathbb{S K}_{m, n} \longrightarrow \mathbb{S K}_{m, n+1}$ by the rule

$$
S([a, \mathfrak{g}, n])=(-1)^{|\mathfrak{g}|} \begin{cases}{[a, \mathfrak{g}, n+1],} & \text { if } n \text { is even, } \\ -\sum_{i=1}^{q-2} i\left[\gamma^{i} a, \mathfrak{g}, n+1\right], & \text { if } n \text { is odd, }\end{cases}
$$

and define the diagonal shift operator $\Delta_{\mathfrak{p}}: \mathbb{S K}_{m, n} \longrightarrow \mathbb{S K}_{m-1, n+2}$ associated with a prime $\mathfrak{p}$ by the rule:

$$
\Delta_{\mathfrak{p}}([a, \mathfrak{g}, n])=0, \quad \text { if } \mathfrak{p} \nmid \mathfrak{g},
$$

and if $\mathfrak{g}=\mathfrak{p}_{1} \mathfrak{p}_{2} \cdots \mathfrak{p}_{m}$ with $\mathfrak{p}_{1}<\mathfrak{p}_{2}<\cdots<\mathfrak{p}_{m}$,

$$
\Delta_{\mathfrak{p}_{r}}([a, \mathfrak{g}, n])=(-1)^{r}\left[a, \mathfrak{g} / \mathfrak{p}_{r}, n+2\right] .
$$

The reader can check directly the following lemma, or refer to [Da, Thms. 4-5].

Lemma 3. i) $S \delta+\delta S=q-1$ and $\partial S+S \partial=0$. Thus $(\partial+\delta) S+S(\partial+\delta)=q-1$.

ii) $\partial \Delta_{\mathfrak{p}}=\Delta_{\mathfrak{p}} \partial$ and $\delta \Delta_{\mathfrak{p}}=\Delta_{\mathfrak{p}} \delta$.

Given a canonical basis class $[0, \mathfrak{g},-n]$ with $|\mathfrak{g}|=n$ even, one can construct a representing cycle

$$
C=\bigoplus_{i=0}^{n} C_{i,-i}, \quad C_{n,-n}=[0, \mathfrak{g},-n]
$$

such that $C_{i,-i}=\sum n_{j}\left[a_{j}, \mathfrak{g}_{j},-i\right]$ with $\operatorname{sgn}\left(a_{j}\right)=1$ for $i$ odd, $\operatorname{sgn}\left(a_{j}\right) \neq \gamma^{q-2}$ for $i$ even, and no term of the form $[0, \mathfrak{h},-m]$ except $[0, \mathfrak{g},-n]$ occurs, as follows. 
Suppose that one has constructed $C_{i,-i}$. If $\partial C_{i,-i}=\sum_{j} m_{j}\left[a_{j}, \mathfrak{g}_{j},-i\right]$, then

$$
C_{i-1,1-i}=(-1)^{i-1} \begin{cases}\sum_{j} m_{j}\left\langle a_{j}\right\rangle\left[a_{j}, \mathfrak{g}, 1-i\right], & \text { if } i \text { is even, } \\ \sum_{j} m_{j} \sum_{k \geq 0}^{\kappa\left(a_{j}\right)-1}\left[\gamma^{k-\kappa\left(a_{j}\right)} a_{j}, \mathfrak{g}, 1-i\right], & \text { if } i \text { is odd, }\end{cases}
$$

where $\operatorname{sgn}\left(a_{j}\right)=\gamma^{\kappa\left(a_{j}\right)}$ with $0 \leq \kappa\left(a_{j}\right)<q-1$.

We call $C$ a semi-canonical lifting. Such a construction also works for canonical basis classes of $H^{1}$ and for the boundary elements of $\mathbb{S K}$.

For an element $C$ of $\mathbb{S K}$ and a square-free monic polynomial $\mathfrak{g}$, we let $C^{\{\mathfrak{g}\}}$ be the $\mathfrak{g}$-component, i.e., the part that includes those of the form $[*, \mathfrak{g}, *]$. Following the same lines as Proposition 7 of [Da], we have:

Proposition 4. Let $C=\bigoplus_{i+j=\ell} C_{i, j}$ be a cycle in $\mathbb{S K}$. For a fixed monic squarefree polynomial $\mathfrak{g}$, write $C_{k, \ell-k}^{\{\mathfrak{g}\}}=\sum n_{i}\left[a_{i}, \mathfrak{g}, \ell-k\right]$. Then we have

$$
\sum n_{i}\left[a_{i}\right] \in \begin{cases}H^{1}(J, \mathbb{U}) & \text { if } \ell-k \text { is odd } \\ H^{2}(J, \mathbb{U}) & \text { if } \ell-k \text { is even. }\end{cases}
$$

The assertions in the next proposition are the analogues of Theorem 8 and Propositions 3 and 4 in Da]. The ideas of the proof are taken from there.

Proposition 5. Let $\mathbf{a}=\sum m_{i}\left[a_{i}\right] \in H^{2}(J, \mathbb{U})$. We have

i) For each $\mathfrak{t} \in(A / \mathfrak{f})^{*}, \sum m_{i}\left\langle a_{i}\right\rangle=\sum m_{i}\left\langle\mathfrak{t} a_{i}\right\rangle$.

ii) Let $m$ be the coefficient of [0] in $\mathbf{a}$. Then $q-1$ divides $\sum m_{i}-m$.

iii) Let $C$ be a cycle in $\mathbb{S K}$ such that $C=\bigoplus_{i+j=0} C_{i, j}, C_{0,0}=\sum m_{i}\left[a_{i}, 1,0\right]$, and $(\partial+\delta) C=0$. Let further

$$
C_{1,-1}=\sum n_{j}\left[b_{j}, \mathfrak{p}_{j},-1\right] .
$$

Then $\sum n_{j} \operatorname{deg} \mathfrak{p}_{j} \equiv 0 \bmod (q-1)$.

\section{Algebraic gamma monomials}

Thakur (Th) defined some $\Gamma$-function in characteristic $p$. We change Thakur's definition slightly by the formula

$$
\Gamma(z)=\prod_{\mathfrak{a} \in A_{+}}\left(1+\frac{z}{\mathfrak{a}}\right)^{-1},
$$

where $A_{+}$is the set of all monic polynomials in $A$. This $\Gamma(z)$ is just the $\Pi(z)$ of Thakur. Let $\tilde{\pi}$ denote the fundamental period of the Carlitz module, which is unique up to a factor of $\mathbb{F}_{q}^{*}$. Let $e=e_{C}$ be the Carlitz exponential. The $\Gamma$-function has the following nice properties.

Theorem 6 ([Th], Theorem 6.1.1, Theorem 6.2.1). (1) Reflection formula:

$$
\prod_{\theta \in J} \Gamma(\theta z)=\frac{\tilde{\pi} z}{e(\tilde{\pi} z)} .
$$

(2) Multiplication formula: For $\mathfrak{f} \in A_{+}$of degree $d$ we have

$$
\prod_{\substack{\mathfrak{a} \in A \\ \operatorname{deg} \mathfrak{a}<d}} \Gamma\left(\frac{z+\mathfrak{a}}{\mathfrak{f}}\right)=\tilde{\pi}^{\left(q^{d}-1\right) /(q-1)}\left((-1)^{d} \mathfrak{f}\right)^{q^{d} /(1-q)} R_{d}(z) \Gamma(z),
$$

where $R_{d}(z)=\prod_{\substack{\mathfrak{a} \text { monic } \\ \operatorname{deg} \mathfrak{a}<d}}(z+\mathfrak{a})$. 
For $a \in K / A$ we denote by $\{a\}$ the representative of $a$ such that $|a|_{\infty}<1$, where $\|_{\infty}$ is the absolute value at $\infty=\left(\frac{1}{T}\right)$. For each $\mathbf{a}=\sum m_{i}\left[a_{i}\right] \in \mathcal{A}$, we define the $\Gamma$-monomial, $e$-monomial, and $r$-monomial, respectively, by

$$
\begin{gathered}
\Gamma(\mathbf{a})=\tilde{\pi}^{\frac{T S(\mathbf{a})}{q-1}} \prod \Gamma\left(\left\{a_{i}\right\}\right)^{-m_{i}}, \\
e(\mathbf{a})=\prod_{a_{i} \neq 0} e\left(\tilde{\pi} a_{i}\right)^{m_{i}}, \quad \text { and } \quad r(\mathbf{a})=\prod_{a_{i} \neq 0}\left\{a_{i}\right\}^{m_{i}} .
\end{gathered}
$$

By abuse of notation, we also write $\Gamma\left(\sum n_{i}\left[a_{i}, *, *\right]\right)$ to mean $\Gamma\left(\sum n_{i}\left[a_{i}\right]\right)$. This notation will also be applied to $e$ - and $r$-monomials. In what follows $a \in K / A$ always means that $a=\{a\}$ unless otherwise stated. It is known that $\Gamma(\mathbf{a})$ is algebraic over $K$ if $\mathbf{a}=\sum m_{i}\left[a_{i}\right] \in H^{2}(J, \mathbb{U})$. In fact, we have shown that $\Gamma(\mathbf{a})^{q-1}=\sqrt[q-1]{r} e(\mathbf{a})$ for some $r \in K^{*}$; see [BGY] Thm. 7.2]. Using the double complex, we can express $r$ explicitly. Let $C=\bigoplus_{i+j=0} C_{i, j}$ be a cycle in $\mathbb{S K}$ such that $C_{0,0}=\sum m_{i}\left[a_{i}, 1,0\right]$ and $(\partial+\delta) C=0$. Then $(\partial+\delta) S C=(q-1) C$ and $(q-1) C_{0,0}=\delta S C_{0,0}+\partial S C_{1,-1}$. Note that $\Gamma\left((q-1) C_{0,0}\right)=\Gamma(\mathbf{a})^{q-1}$ and $\Gamma\left(\delta S C_{0,0}\right)=e(\mathbf{a}) / r(\mathbf{a})$. We get

$$
\Gamma(\mathbf{a})^{q-1}=\frac{\Gamma\left(\partial S C_{1,-1}\right)}{r(\mathbf{a})} e(\mathbf{a}) .
$$

Now the following Kummer property of $\Gamma$-monomials, which originally is due to $\operatorname{Sinha}([\mathrm{Si}])$, is a direct result of the equality. We denote by $K_{\mathfrak{f}}$ the cyclotomic function field of conductor $\mathfrak{f}$.

Theorem 7. Let $\mathbf{a}=\sum m_{i}\left[a_{i}\right] \in H^{2}(J, \mathbb{U})$ and let $\mathfrak{f}$ be the least common multiple of the denominators of $\mathbf{a}$. Then $K_{\mathfrak{f}}(\Gamma(\mathbf{a})) / K_{\mathfrak{f}}$ is a Kummer extension.

Proof. For any irreducible polynomial $\mathfrak{p}$ with degree $d$, we have

$$
\partial S[b, \mathfrak{p},-1]=\sum_{i=1}^{q-2} i\left(\left[\gamma^{i} b, 1,0\right]-\sum_{\operatorname{deg} \mathfrak{u}<d}\left[\frac{\gamma^{i} b+\mathfrak{u}}{\mathfrak{p}}, 1,0\right]\right)
$$

and thus

$$
\Gamma(\partial S[b, \mathfrak{p},-1])=\left((-1)^{d} \mathfrak{p}\right)^{\frac{q^{d}(q-2)}{2}} \cdot\left(\prod_{i=1}^{q-2} R_{d}\left(\gamma^{i}\{b\}\right)^{-i}\right) .
$$

If $2 \mid q$, then $\Gamma(\partial S[b, \mathfrak{p},-1]) \in K$. If $2 \nmid q$, since $\sqrt[q-1]{(-1)^{d} \mathfrak{p}} \in K_{\mathfrak{p}}$, we get the result by $\operatorname{Eq}(2.1)$.

The key point in the study of $\Gamma$-monomials by means of the double complex is that the factor $\Gamma\left(\partial S C_{1,-1}\right)$ in $(2.1)$ is very simple, if $C$ is a canonically lifted cycle, and for a general cycle $C$ we get information about that factor using homological algebra. In fact, we have the following theorem and corollary, the lines of proof of which are again taken from [Da, Sect. 9].

Theorem 8. Let $n$ be an even positive integer. Let $C=\bigoplus C_{i,-i}$ be the semicanonically lifted cycle from the basis class $[0, \mathfrak{g},-n]$, where $\mathfrak{g}$ is a square-free monic polynomial divisible by $n$ irreducible polynomials. Let $\mathbf{a}=\sum m_{i}\left[a_{i}\right]$, where $C_{0,0}=$ $\sum m_{i}\left[a_{i}, 1,0\right]$. Then $\Gamma(\mathbf{a})^{q-1} \in K_{\mathfrak{g}}$. Furthermore,

i) If $\mathfrak{g}=\mathfrak{p q}$ with $d=\operatorname{deg} \mathfrak{p}$ and $e=\operatorname{deg} \mathfrak{q}$, then

$$
\Gamma(\mathbf{a})^{q-1} \equiv \sqrt{\frac{\mathfrak{q}^{d}}{\mathfrak{p}^{e}}} e(\mathbf{a}) \quad \bmod K^{*}
$$


ii) If $n \geq 4$, then

$$
\Gamma\left(\partial S C_{1,-1}\right) \in K^{*} \quad \text { and } \quad \Gamma(\mathbf{a})^{q-1} \equiv e(\mathbf{a}) \bmod K^{*} .
$$

Corollary. Let $n \geq 4$ be an even integer. Let $\mathbf{a}=\sum m_{i}\left[a_{i}\right]$ represent the basis class $[0, \mathfrak{g},-n]$ with $|\mathfrak{g}|=n$, not necessarily a semi-canonical representative. Then

$$
\Gamma(\mathbf{a})^{q-1} \equiv e(\mathbf{a}) \quad \bmod K^{*} .
$$

The following result is an analogue of Theorem 11 in Da.

Proposition 9. Let $\mathbf{a}=\sum m_{i}\left[a_{i}\right] \in H^{2}(J, \mathbb{U})$. Then, with notation as in Proposition 5, we have

$$
\frac{e(\mathbf{a})}{e\left(\mathbf{a}^{\mathfrak{t}}\right)}=\theta_{\mathbf{t}} e(\mathbf{b})^{(q-1)},
$$

for some $\mathbf{b} \in \mathcal{A}$ and $\theta_{\mathfrak{t}}= \pm 1$.

Proof. Let $C=\bigoplus_{i+j=0} C_{i, j}$ be a cycle in $\mathbb{S} \mathbb{K}$ such that $C_{0,0}=\sum m_{i}\left[a_{i}, 1,0\right]$. Then $C-C^{\mathfrak{t}}$ is a boundary. Let $B=\bigoplus_{i+j=1} B_{i, j}$ be a chain in $\mathbb{S} \mathbb{K}$ such that $(\partial+\delta) B=C-C^{\mathbf{t}}$. We have $\frac{e(\mathbf{a})}{e\left(\mathbf{a}^{\mathfrak{t}}\right)}=e\left(\partial B_{1,0}\right) e\left(\delta B_{0,1}\right)$. Note that

$$
e(\partial[0, \mathfrak{p}, 0])=\mathfrak{p}=\prod_{\substack{\operatorname{deg} \mathfrak{u}<\operatorname{deg} \mathfrak{p} \\ \text { monic }}}(-1)^{\frac{q^{\operatorname{deg} \mathfrak{p}}-1}{q-1}} e\left(\frac{\mathfrak{u}}{\mathfrak{p}}\right)^{q-1},
$$

and

$$
e(\partial[a, \mathfrak{p}, 0])=1, \quad \text { if } a \neq 0,
$$

and that $e(\delta[a, 1,1])=-e(a)^{q-1}$. We get the result.

Remark. As the referee points out, if one defines

$$
\sin a=\sqrt[q-1]{-1} \cdot e(\{a\} / \operatorname{sgn}\{a\})
$$

for $a \in K \backslash \mathbb{A}$, then, by making the obvious definition of $\sin \mathbf{a}$, one has

$$
\frac{\sin \mathbf{a}}{\sin \mathbf{a}^{\mathfrak{t}}}=(\sin \mathbf{b})^{q-1},
$$

in strict analogy with Theorem 11 of [Da].

Example. Let $q=3, \mathbf{a}=\left[\frac{1}{T+1}\right]-\left[\frac{T-1}{T(T+1)}\right]$, and $\mathfrak{t}=-T+1$. Then

$$
\frac{e(\mathbf{a})}{e\left(\mathbf{a}^{\mathfrak{t}}\right)}=\frac{e\left(\tilde{\pi} \frac{1}{T(T+1)}\right)}{e\left(\tilde{\pi} \frac{T-1}{T(T+1)}\right)}=-e\left(\tilde{\pi} \frac{1}{T(T+1)}\right)^{2},
$$

since $\lambda=e\left(\tilde{\pi} \frac{1}{T(T+1)}\right)$ satisfies the relation $\lambda^{4}+(T+1) \lambda^{2}+1=0$.

If $\mathfrak{t}=-1$, then

$$
\frac{e(\mathbf{a})}{e\left(\mathbf{a}^{\mathfrak{t}}\right)}=1=e(\mathbf{0})^{2}
$$

Thus $\theta_{\mathfrak{t}}$ changes as $\mathfrak{t}$ varies.

Theorem 10. Let $\mathbf{a}=\sum m_{i}\left[a_{i}\right] \in H^{2}(J, \mathbb{U})$. Let $\mathfrak{f}$ be the least common multiple of the denominators of the $a_{i}$ and let $\mathfrak{t} \in(A / \mathfrak{f})^{*}$. Then

$$
\frac{\Gamma(\mathbf{a})}{\Gamma\left(\mathbf{a}^{\mathfrak{t}}\right)} \in K_{\mathfrak{f}} .
$$


Proof. With notation as in the proof of Proposition 9, let $B_{1,0}=\sum \ell_{j}\left[c_{j}, \mathfrak{p}_{j}, 0\right]$. We may assume that $B$ is a semi-canonically lifted chain. Then the denominators of $c_{j}$ divide $\mathfrak{f}$. From the proof of Proposition 9, it suffices to show that $\Gamma\left(\partial B_{1,0}\right) \in K_{\mathfrak{f}}$. It can be easily checked that

$$
\Gamma\left(\partial B_{1,0}\right) \equiv(-1)^{\frac{\sum \ell_{j} \operatorname{deg} \mathfrak{p}_{j}}{q-1}} \prod \mathfrak{p}_{j}^{\frac{\ell_{j}}{q-1}} \bmod K_{\mathfrak{f}}^{*} .
$$

Thus the result follows.

Similarly, if a and $\mathbf{a}^{\prime}$ represent the same class in $H^{2}(J, \mathbb{U})$, then $\frac{\Gamma(\mathbf{a})}{\Gamma\left(\mathbf{a}^{\prime}\right)} \in K_{\mathfrak{f}}$.

\section{CRiterion For $H^{1}(J, \mathbb{U})$}

The following conclusion is shown in BGY, Cor. 4.2].

Lemma 11. Let $\mathbf{b}=\sum m_{i}\left[b_{i}\right] \in H^{1}(J, \mathbb{U})$. Then for all $i, b_{i} \neq 0$.

Lemma 12. Let $\mathbf{b}=\sum m_{i}\left[b_{i}\right] \in H^{1}(J, \mathbb{U})$, representing any canonical basis class of $H^{1}(J, \mathbb{U})$ indexed by a monic square-free polynomial divisible by at least three primes. Let $C=\bigoplus_{i+j=1} C_{i, j}$ be a cycle such that $C_{0,1}=\sum m_{i}\left[b_{i}, 1,1\right]$. Assume that no term of the form $[0, \mathfrak{p}, 0]$ appears in $C_{1,0}$. Then

$$
\sum m_{i} \equiv 0 \quad \bmod (q-1) \text {. }
$$

Proof. Note that $I S(\partial[b, p, 0])=\frac{q^{\operatorname{deg} p}-1}{q-1} \equiv \operatorname{deg} \mathfrak{p} \bmod (q-1)$ for $b \notin A$. Now follow the proof of Proposition 13 of [Da].

It is shown in [BGY] that $\mathbf{b} \in H^{1}(J, \mathbb{U})$ if and only if $|r(\mathbf{b})|_{\infty}=\left|r\left(\mathbf{b}^{\mathfrak{t}}\right)\right|_{\infty}$ for any $\mathfrak{t} \in(A / \mathfrak{f})^{*}$, where $\mathfrak{f}$ is the least common multiple of the denominators of the $b_{i}$. Here we give another proof of the necessity of this using the double complex. In this way one can get some more information about the $e$-monomials.

Theorem 13. Let $\mathbf{b}=\sum m_{i}\left[b_{i}\right] \in H^{1}(J, \mathbb{U})$ and let $\mathfrak{f}$ be the least common multiple of the denominators of the $b_{i}$. Then

$$
|r(\mathbf{b})|_{\infty}=\left|r\left(\mathbf{b}^{\mathfrak{t}}\right)\right|_{\infty},
$$

for all $\mathfrak{t} \in(A / \mathfrak{f})^{*}$. Furthermore, we have more information about e-monomials in the following special cases:

First case: If $\mathbf{b}$ represents a canonical basis class of $H^{1}(J, \mathbb{U})$, indexed by a single irreducible polynomial $\mathfrak{p}$, then $e(\mathbf{b})^{(q-1)}=e(\mathbf{b})^{(q-1)}=\sqrt{ \pm \mathfrak{p}}$.

Second case: Let $\mathbf{b}$ represent a canonical basis class of $H^{1}(J, \mathbb{U})$ indexed by a monic square-free polynomial divisible by at least three primes. Let $C=\bigoplus C_{i,-i+1}$ be a cycle such that $C_{0,1}=\sum m_{i}\left[b_{i}, 1,1\right]$. Assume that no term of the form $[0, \mathfrak{p}, 0]$ appears in $C_{1,0}$. Then $e\left(\mathbf{b}^{\mathfrak{t}}\right) \in \mathbb{F}_{q}^{*}$ for any $\mathfrak{t} \in(A / \mathfrak{f})^{*}$.

Proof. The first statement follows by linearity from the two special cases, since $|r(\mathbf{b})|_{\infty}=|e(\mathbf{b})|_{\infty}$. Let $C=\bigoplus C_{i,-i+1}$ be a cycle such that $C_{0,1}=\sum m_{i}\left[b_{i}, 1,1\right]$.

First Case: Let $C_{1,0}=[0, \mathfrak{p}, 0]$. Then we know that $e\left(-\partial C_{1,0}\right)=e\left(-\partial C_{1,0}^{\mathrm{t}}\right)=\mathfrak{p}$. Thus

$$
e\left(\delta C_{0,1}\right)=e\left(\delta C_{0,1}^{\mathfrak{t}}\right)=\mathfrak{p} .
$$

However, $e\left(\delta C_{0,1}\right)=(-1)^{\sum m_{i}} e(\mathbf{b})^{q-1}$ and $e\left(\delta C_{0,1}^{\mathfrak{t}}\right)=(-1)^{\sum m_{i}} e\left(\mathbf{b}^{\mathfrak{t}}\right)^{q-1}$. Then the result follows from the fact that $\sigma_{\mathfrak{t}} e(\mathbf{b})=e\left(\mathbf{b}^{\mathfrak{t}}\right)$.

Second Case: In this case, $e\left(\partial C_{1,0}\right)=1$. Then the result follows in the same way as the first case, by using Lemma 11. 
The following proposition is a direct consequence of Theorem 13.

Proposition 14. Let $\mathbf{b}=\sum m_{i}\left[b_{i}\right] \in H^{1}(J, \mathbb{U})$. Assume that $\mathbf{b}$ represents a canonical basis class of $H^{1}(J, \mathbb{U})$, indexed by monic square-free polynomials divisible by at least three monic irreducibles. Let $C$ be a cycle in $\mathbb{S K}$ such that $C=\bigoplus_{i+j=0} C_{i, j}$, $C_{0,0}=\sum m_{i}\left[b_{i}, 1,0\right]$ and $(\partial+\delta) C=0$. Assume that no term of the form $[0, \mathfrak{p},-1]$ appears in $C_{1,-1}$. Then for each $\mathfrak{t}$ coprime to the least common multiple of the denominators of the $b_{i}$, we have

$$
\prod_{i} \operatorname{sgn}\left(\left\{b_{i} \mathfrak{t}\right\}\right)^{m_{i}}=\prod_{i} \operatorname{sgn}\left(\left\{b_{i}\right\}\right)^{m_{i}} .
$$

Proof. From Theorem 13, we know that $e(\mathbf{b}) \in K^{*}$. Thus $e\left(\mathbf{b}^{\mathfrak{t}}\right)=\sigma_{\mathfrak{t}} e(\mathbf{b})=e(\mathbf{b})$ and, further, $\operatorname{sgn}(e(\mathbf{b}))=\operatorname{sgn}\left(e\left(\mathbf{b}^{\mathfrak{t}}\right)\right)$. Since $\sum m_{i} \equiv 0 \bmod (q-1)$ from Lemma 12 , we get the result.

\section{Galois properties of $K_{\mathfrak{f}}(\Gamma(\mathbf{a})) / K$}

Let $\mathbf{a}=\sum m_{i}\left[a_{i}\right] \in H^{2}(J, \mathbb{U})$, and let $\mathfrak{f}$ be the least common multiple of the denominators of the $a_{i}$. In this section we consider the extension $K_{\mathfrak{f}}(\Gamma(\mathbf{a}))$ over $K$. Let $C=\bigoplus C_{i,-i}$ be a cycle in $\mathbb{S K}$ such that $C_{0,0}=\sum m_{i}\left[a_{i}, 1,0\right]$. Write

$$
v=\Gamma\left(\partial S C_{1,-1}\right) \text { and } \Gamma(\mathbf{a})^{q-1}=v e(\mathbf{a}) / r(\mathbf{a}) .
$$

Let $\sigma$ be an element of $\operatorname{Gal}(\bar{K} / K)$ whose restriction to $K_{\mathfrak{f}}$ is $\sigma_{\mathfrak{t}}$, where $\bar{K}$ is the separable closure of $K$. Then

$$
\left(\frac{\Gamma(\mathbf{a})}{\sigma \Gamma(\mathbf{a})}\right)^{q-1}=\frac{v}{\sigma v} \theta_{\mathrm{t}} e(\mathbf{b})^{q-1},
$$

where $\theta_{\mathfrak{t}}$ and $\mathbf{b}$ are given in Proposition 9 . Hence

$$
\frac{\Gamma(\mathbf{a})}{\sigma \Gamma(\mathbf{a})} \in K_{\mathfrak{f}} \Longleftrightarrow \frac{v}{\sigma v} \theta_{\mathfrak{t}}=1
$$

When is $K_{\mathfrak{f}}(\Gamma(\mathbf{a})) / K$ a Galois extension? The following theorems are the main results of the paper.

Theorem 15. Assume $q$ is odd. Let $\mathbf{a}=\sum m_{i}\left[a_{i}\right] \in H^{2}(J, \mathbb{U})$, and let $\mathfrak{f}$ be the least common multiple of the denominators of the $a_{i}$. Then $K_{\mathfrak{f}}(\Gamma(\mathbf{a}))$ is a Galois extension of $K$.

In the number field case, Das first shows the analogue of Theorem $8^{\prime}$ Da Thm. $15(\mathrm{C})]$ and then derives easily his main result [Da, Thm. 16]. In our case, Theorem $8^{\prime}$ is hard to prove because of the extra factor in the functional equation of the $\Gamma$ function. So we first show this theorem and then derive Theorem $8^{\prime}$. In the following proof we omit some complicated computations as suggested by the referee, because these are not interesting and do not offer any deeper insights.

Proof. The notation is as above. Using the analogue of [Da, Prop. 16] we can assume that the cycle $C$ that a represents is semi-canonically lifted from a basis class. We know from Proposition 5 that $e(\mathbf{a})$ and $e\left(\mathbf{a}^{\mathfrak{t}}\right)$ lie in $K_{\mathfrak{f}}^{+}$, the maximal real subfield of $K_{\mathfrak{f}}$. Hence the signs of $e(\mathbf{a})$ and $e\left(\mathbf{a}^{\mathfrak{t}}\right)$ make sense. It is not hard to get $\operatorname{sgn}\left(e(\mathbf{a}) / e\left(\mathbf{a}^{\mathfrak{t}}\right)\right)=1$. 
Let $B$ be a semi-canonically lifted chain such that $C-C^{\mathfrak{t}}=(\partial+\delta) B$. Let $B_{0,1}=\sum n_{j}\left[b_{j}, 1,1\right]$ and let $\mathbf{b}=\sum n_{j}\left[b_{j}\right]$. We see that $\operatorname{sgn}\left(e(\mathbf{b})^{q-1}\right)=(-1)^{\sum n_{j}}$ and thus $\theta_{\mathfrak{t}}=(-1)^{\sum n_{j}}$ by Proposition 9 .

Write $B_{1,0}=\sum l_{k}\left[c_{k}, \mathfrak{p}_{k}, 0\right]$. Using the facts that $\partial B_{1,0}+\delta B_{0,1}=C_{0,0}-C_{0,0}^{\mathrm{t}}$ and $I S\left(C_{0,0}-C_{0,0}^{\mathfrak{t}}\right)=0$, we see that

$$
\sum l_{k} \operatorname{deg} \mathfrak{p}_{k} \equiv \sum n_{j} \bmod 2
$$

Since $\operatorname{sgn}\left(\Gamma(\partial[b, \mathfrak{p}, 0])^{q-1}\right)=(-1)^{\operatorname{deg} \mathfrak{p}}$, we get

$$
\operatorname{sgn}\left(\Gamma\left(\partial B_{1,0}\right)^{q-1}\right)=(-1)^{\sum l_{k} \operatorname{deg} \mathfrak{p}_{k}}=(-1)^{\sum n_{j}}=\theta_{\mathfrak{t}} .
$$

So we need to relate the sign of $\left.\Gamma\left(\partial B_{1,0}\right)^{q-1}\right)$ to $v / \sigma_{\mathfrak{t}} v$, for which we consider two cases separately.

First Case: a represents the basis class $[0, \mathfrak{p q},-2]$. Write $d_{\mathfrak{p}}=\operatorname{deg} \mathfrak{p}$ and $N_{\mathfrak{p}}=\#\left\{\mathfrak{a}:\right.$ monic, $\left.d_{\mathfrak{a}}<d_{\mathfrak{p}}, \operatorname{sgn}\left(\left\{\frac{\mathfrak{t a}}{\mathfrak{p}}\right\}\right) \notin \mathbb{F}_{q}^{* 2}\right\}$. Using Theorem 8 and the analogue of the classical Gauss lemma, we get by direct calculation

$$
\frac{v}{\sigma v}=(-1)^{d_{\mathfrak{p}} N_{\mathfrak{q}}+d_{\mathfrak{q}} N_{\mathfrak{p}}}=\operatorname{sgn}\left(\Gamma\left(\partial B_{1,0}\right)^{q-1}\right) .
$$

Thus we have the result in the first case.

Second Case: a represents a canonical basis class indexed by a squarefree polynomial $\mathfrak{g}$ divisible by at least four distinct irreducibles. In this case $v \in K$ by Theorem 8, and so $\frac{v}{\sigma v}=1$. We define some operators on $\mathbb{S} \mathbb{K}$ as follows.

$$
\begin{aligned}
& \mathfrak{t}:[a, *, *] \mapsto\left[a^{\mathfrak{t}}, *, *\right], \\
& I:[a, *, k] \mapsto\langle a\rangle[a, *, k-1] \quad \text { for } k \text { odd, } \\
& J:[a, *, k] \mapsto \sum_{l=0}^{\kappa(a)-1}\left[\gamma^{l-\kappa(a)} a, *, k-1\right] \quad \text { for } k \text { even, }
\end{aligned}
$$

where $\kappa(a)$ is defined by $\operatorname{sgn}(a)=\gamma^{\kappa(a)}$ with $0 \leq \kappa(a)<q-1$. Note that $J I=0$.

Let $C$ be the cycle obtained by the semi-canonical lifting of $k_{\mathfrak{g}}$. Our aim is to compute $\operatorname{sgn}\left(\Gamma\left(\partial B_{1,0}\right)^{q-1}\right)=(-1)^{\sum l_{k} \operatorname{deg} \mathfrak{p}_{k}}$, where $B_{1,0}=\sum l_{k}\left[c_{k}, \mathfrak{p}_{k}, 0\right]$. So we only need to consider the parities of the total sum of $B_{1,0}^{\left\{\mathfrak{p}_{k}\right\}}$. Let $C_{n,-n}=[0, \mathfrak{g},-n]$. Then we have

$$
B_{1,0}=(J \partial I t-J t I \partial) C_{2,-3}+J \partial I J \partial E+J \partial I \partial J F,
$$

for some chains $E, F \in \mathbb{S} \mathbb{K}$. A straightforward but tedious computation shows that $T S\left(B_{1,0}^{\{\mathfrak{p}\}}\right) \equiv 0 \bmod (q-1)$. Then $\operatorname{sgn}\left(\Gamma\left(\partial B_{1,0}\right)^{q-1}\right)=1$, which implies the result. Lemma 12 and Proposition 14 are used in the course of the computation.

We know from Proposition 9 that $\mathbb{F} K_{\mathfrak{f}}(\sqrt[q-1]{e(\mathbf{a})})$ is a Galois extension of $K$, where $\mathbb{F}$ is the quadratic extension of $\mathbb{F}_{q}$. But in the second case we get more.

Theorem 16. Let a $\in H^{2}(J, \mathbb{U})$ represent a canonical basis class indexed by a squarefree polynomial $\mathfrak{f}$ divisible by at least four distinct irreducibles. Then $K_{\mathfrak{f}}(\sqrt[q-1]{e(\mathbf{a})})$ is Galois over $K$.

Proof. Since $\frac{e(\mathbf{a})}{\sigma_{\mathfrak{t}} e(\mathbf{a})}=\frac{e(\mathbf{a})}{e\left(\mathbf{a}^{\mathfrak{t}}\right)}=\theta_{\mathfrak{t}} e(\mathbf{b})^{q-1}$, for some $\mathbf{b}, K_{\mathfrak{f}}(\sqrt[q-1]{e(\mathbf{a})})$ is Galois over $K$ if and only if $\theta_{\mathfrak{t}}=1$. Thus the result follows from the proof of Theorem 15 . 
We have seen in the proof of Theorem 15 that if a represents a canonical basis class indexed by a square-free polynomial $\mathfrak{g}$ divisible by at least four irreducibles, then the total degree of $B_{1,0}^{\{\mathfrak{p}\}}$ is divisible by $q-1$ for any prime $\mathfrak{p}$ and $\theta_{\mathfrak{t}}=1$. Hence $\Gamma\left(\partial B_{1,0}\right) \in K^{*}$. From the proof of Proposition 9, we have

$$
\left(\frac{\Gamma(\mathbf{a})}{\Gamma\left(\mathbf{a}^{\mathfrak{t}}\right)}\right)^{q-1}=\Gamma\left(\partial B_{1,0}\right)^{q-1} \Gamma\left(\delta B_{0,1}\right)^{q-1}=\Gamma\left(\partial B_{1,0}\right)^{q-1} r\left(B_{0,1}\right)^{1-q} \theta_{\mathfrak{t}} \frac{e(\mathbf{a})}{e\left(\mathbf{a}^{\mathfrak{t}}\right)} .
$$

Finally, we have the following stronger version of Theorem 8 .

Theorem $\mathbf{8}^{\prime}$. Let $n \geq 4$ be an even positive integer. Let $C=\bigoplus C_{i,-i}$ be the semi-canonically lifted cycle from the basis class $[0, \mathfrak{g},-n]$, where $\mathfrak{g}$ is a square-free monic polynomial divisible by $n$ irreducible polynomials. Let $\mathbf{a}=\sum m_{i}\left[a_{i}\right]$, where $C_{0,0}=\sum m_{i}\left[a_{i}, 1,0\right]$. Then

$$
\Gamma(\mathbf{a})^{q-1}=r e(\mathbf{a}) \quad \text { and } \quad \Gamma\left(\mathbf{a}^{\mathfrak{t}}\right)^{q-1}=r s^{q-1} e\left(\mathbf{a}^{\mathfrak{t}}\right)
$$

for some $r, s \in K^{*}$.

In $[\mathrm{BY}]$ we proved that if $\mathbf{a} \in H^{2}(J, \mathbb{U})$ represents the basis class $[0, \mathfrak{p q},-2]$, then $K_{\mathfrak{p q}}(\sqrt[q-1]{e(\mathbf{a})})$ is nonabelian over $K$. Here we also give an example where $K_{\mathfrak{f}}(\Gamma(\mathbf{a}))$ is not abelian over $K$.

Example. We assume that $q=3$. We can easily compute that the cycle $C=$ $C_{0,0} \oplus C_{1,-1} \oplus C_{2,-2}$ is the semi-canonically lifted cycle of the canonical basis class $[0, T(T+1),-2]$, where

$$
\begin{gathered}
C_{2,-2}=[0, T(T+1),-2], \\
C_{1,-1}=\left[\frac{1}{T}, T+1,-1\right]-\left[\frac{1}{T+1}, T,-1\right], \\
C_{0,0}=\left[\frac{1}{T+1}, 1,0\right]-\left[\frac{T-1}{T(T+1)}, 1,0\right] .
\end{gathered}
$$

Thus

$$
\mathbf{a}_{T(T+1)}=\left[\frac{1}{T+1}\right]-\left[\frac{T-1}{T(T+1)}\right] .
$$

A simple computation gives

$$
\Gamma\left(\mathbf{a}_{T(T+1)}\right)^{2}=\sqrt{\frac{T}{T+1}} \frac{e\left(\frac{\tilde{\pi}}{T+1}\right)}{e\left(\frac{(T-1) \tilde{\pi}}{T(T+1)}\right)}=u,
$$

using the relation $\Gamma\left(\mathbf{a}_{T(T+1)}\right)^{2}=\Gamma\left(\delta S C_{0,0}\right) \Gamma\left(\partial S C_{1,-1}\right)$. Let $\sigma=\sigma_{T-1}$ and $\tau=$ $\sigma_{-T+1}$. Let $\lambda=e\left(\frac{\tilde{\pi}}{T(T+1)}\right)$. Then we can check that

$$
\frac{u}{\sigma u}=\lambda^{2}, \quad \frac{u}{\tau u}=\lambda^{2}, \quad \text { and } \quad \frac{u}{\sigma \tau u}=1 .
$$

Let

$$
v_{\sigma}=\lambda \quad v_{\tau}=\lambda \quad \text { and } \quad v_{\sigma \tau}=1 .
$$

Let $\tilde{\eta} \in \operatorname{Gal}\left(K_{\mathfrak{f}}(\Gamma(\mathbf{a})) / K\right)$ be the lifting of $\eta \in \operatorname{Gal}\left(K_{\mathfrak{f}} / K\right)$ such that $v_{\eta} \tilde{\eta} \sqrt{u}=\sqrt{u}$. Then using the fact that $\lambda^{4}+(T+1) \lambda^{2}+1=0$, we get $\tilde{\sigma} \tilde{\tau}=-\tilde{\tau} \tilde{\sigma}$ on $\sqrt{u}$.

Remark. It would be very interesting to know whether $K_{\mathfrak{f}}(\Gamma(\mathbf{a}))$ is abelian over $K$, or equivalently by the last theorem, whether $K_{\mathfrak{f}}(\sqrt[q-1]{e(\mathbf{a})})$ is abelian over $K$, if a represents a canonical basis class indexed by a monic square-free polynomial divisible by at least four irreducibles. In the classical case this is verified by Das 
([Da], Theorem 21), with the aid of a theorem of Deligne (Theorem 7.18(b) of [De], Theorem 19 of [Da] $)$, that is,

$$
\sigma\left(\frac{\Gamma(\mathbf{a})}{\tau \Gamma(\mathbf{a})}\right)=\frac{\Gamma\left(\mathbf{a}^{\mathfrak{t}}\right)}{\tau \Gamma\left(\mathbf{a}^{\mathfrak{t}}\right)}
$$

where $\mathbf{a} \in H^{2}(J, \mathbb{U}), \sigma, \tau \in \operatorname{Gal}(\bar{K} / K)$ and the restriction of $\sigma$ on $K_{\mathfrak{f}}$ is $\sigma_{\mathfrak{t}}$. Here

$\bar{K}$ is the separable closure of $K$. Note that it is easy to see that $\frac{\Gamma(\mathbf{a})}{\tau \Gamma(\mathbf{a})} \in K_{\mathfrak{f}}$ by Theorem 8 . We also note that when a represents a semi-canonically lifted cycle from a basis class, then

$$
\sigma\left(\frac{\Gamma(\mathbf{a})}{\tau \Gamma(\mathbf{a})}\right)^{q-1}=\left(\frac{\Gamma\left(\mathbf{a}^{\mathfrak{t}}\right)}{\tau \Gamma\left(\mathbf{a}^{\mathfrak{t}}\right)}\right)^{q-1}
$$

using Theorem $8^{\prime}$.

If one disposes of an analogue of Deligne's theorem above, then one can easily show, with the aid of Theorem $8^{\prime}$ and following the same method as in Da, that $K_{\mathfrak{f}}(\Gamma(\mathbf{a}))$ is abelian over $K$ if a satisfies the above conditions.

Sinha Si] has proven Deligne's reciprocity for function fields (Theorem 7.18(a) of [De]) using Anderson's theory of solitons. Thus one may also use the theory of solitons to prove the analogue of Deligne's theorem (Theorem 7.18(b) of [De]), which is beyond the reach of the present paper. However, we hope that an elementary proof using the double complex may be possible. Anderson's recent work on the epsilon extension yields an elementary method to show that $K(\sqrt[q-1]{e(\mathbf{a})})$ is abelian over $K$.

Let $K^{a b}$ be the maximal abelian extension of $K$ and let $G^{a b}=\operatorname{Gal}\left(K^{a b} / K\right)$. Following Anderson [An2, two of us [BY] defined an injective homomorphism

$$
\mathbf{D}: H^{0}\left(G^{a b}, K^{a b *} / K^{a b *(q-1)}\right) \longrightarrow \bigwedge^{2} H^{1}\left(G^{a b}, \mathbb{Z} /(q-1) \mathbb{Z}\right)
$$

and showed it is an isomorphism. We can also express $\mathbf{D}$ explicitly; see [BY, Sect. 3.5] for detail. The map $\mathbf{D}$ has the property that for $u \in K^{a b *}$,

$$
\mathbf{D}\left(u \bmod K^{a b *(q-1)}\right)=0 \Longleftrightarrow \sqrt[q-1]{u} \in K^{a b} .
$$

This proposes an elementary method for showing that $\sqrt[q-1]{e(\mathbf{a})} \in K^{a b}$ if a satisfies the condition above. But the calculation of $\mathbf{D}(\sqrt[a-1]{e(\mathbf{a})})$ would be too complicated to take. About this question, we refer the reader to Remark 4.4.2 in An2].

\section{ACKNOWLEDGEMEnts}

We are very grateful to the referee for many valuable suggestions, especially, for the definition of the vertical shift operator, which is more effective than our old one and enabled a significant shortening of the paper.

\section{REFERENCES}

[An1] G. Anderson, A double complex for computing the sign-cohomology of the universal ordinary distribution, Contemp. Math. 224 (1999), 1-27. MR 99k:11169

[An2] G. Anderson, Kronecker-Weber plus epsilon, Duke Math. J. 114 (2002), 439-475.

[BGY] S. Bae, E.-U. Gekeler, and L. Yin, Distributions and $\Gamma$-monomials, Math. Ann. 321 (2001), 463-478. MR 2002i:33002

[BY] S. Bae, and L. Yin, Carlitz-Hayes plus Anderson's epsilon, Submitted for publication.

[Da] P. Das, Algebraic gamma monomials and double coverings of cyclotomic fields, Trans. Amer. Math. Soc. 352 (2000), 3557-3594. MR 2000m:11107 
[De] P. Deligne, J. Milne, A. Ogus, and K. Shih, Hodge cycles, Motives, and Shimura Varieties, Lecture Notes in Math. 900 (1982). MR 84m:14046

[GR] S. Galovich and M. Rosen, Distributions on rational function fields, Math. Ann. 256 (1981), 549-560. MR 83e:12007

[Ha] D. Hayes, Explicit class field theory for rational function fields, Trans. Amer. Math. Soc. 189 (1974), 77-91. MR 48:8444

[Ou] Y. Ouyang, The group cohomology of the universal ordinary distribution, J. reine angew. Math. 537 (2001), 1-32. MR 2002f:11148

[Si] S. Sinha, Deligne's reciprocity for function fields, J. Number Theory 63 (1997), 65-88. MR 98a:11074

[Th] D. Thakur, Gamma functions for function fields and Drinfeld modules, Ann. of Math. 134 (1991), 25-64. MR 92g:11058

Department of Mathematics, KAist, Taejon 305-701, Korea

E-mail address: shbae@math.kaist.ac.kr

Department of Mathematics, Saarland University, D-66041 Saarbrucken, Germany

E-mail address: gekeler@math.uni-sb.de

Department of Mathematics, Chungnam National University, Taejon 305-764, Korea

E-mail address: plkang@math.cnu.ac.kr

Department of Mathematical Sciences, Tsinghua University, Beijing 100084, People's Republic of China

E-mail address: lsyin@math.tsinghua.edu.cn 